\title{
El papel de los servicios bibliotecarios en línea en la formación universitaria a distancia en la Argentina*
}

\author{
Gustavo Liberatore \\ Dpto. Documentación. \\ Universidad Nacional de Mar del Plata. \\ E-mail: gliberat@mdp.edu.ar \\ Andrés Vuotto \\ Dpto. Documentación. \\ Universidad Nacional de Mar del Plata. \\ E-mail: avuotto@mdp.edu.ar
}

\section{Resumen}

Se lleva a cabo un relevamiento de la situación de los servicios bibliotecarios en línea en los programas de educación a distancia (EAD) de las universidades públicas argentinas, con objeto de evaluar su desarrollo y grados de integración a estas ofertas educativas. El estudio se realiza dentro del entorno web a partir del análisis de las interfaces que presentan los sistemas de EAD.

\section{Palabras clave}

Servicios bibliotecarios en línea; Educación a distancia; Argentina.

The role of the online library services for distance education in Argentina

\begin{abstract}
This is a report about the situation of the online library services concerning distance education programs carried out in public universities of Argentina for the purpose of evaluating their development and integration of the educational offers. This study is carried out within the web environment, starting with the analysis of the interfaces that present the systems of distance education.
\end{abstract}

Keywords

Services on-line librarians; Distance education; Argentina.

"Una versión preliminar de este trabajo fue presentada en el 70을 Congreso de IFLA, Buenos Aires, 22-27 de agosto de 2004.

\section{INTRODUCCIÓN}

La existencia de la educación a distancia (en adelante EAD) en cualquiera de los niveles formativos se remonta a muchas décadas atrás, teniendo sus orígenes en Estados Unidos y Europa. Estos sistemas de educación no presencial fueron creados, en principio, para salvar las barreras geográficas y extender la oferta educativa a zonas o regiones alejadas de los principales centros formativos. Como consecuencia de ello, la EAD siempre estuvo condicionada por los medios de comunicación presentes en cada época para hacer llegar sus servicios. En la actualidad, las tecnologías de la información y comunicación (TIC) han revolucionado el ámbito de la EAD, teniendo como espacio de desarrollo y aplicación a la red Internet (Levis y Gutiérrez, 2000).

Sin embargo, a pesar de la gran evolución de estas tecnologías, los sistemas de EAD siguen basando su estructura en tres componentes principales: cuerpo docente, materiales didácticos y material bibliográfico. Estos tres elementos definen, independientemente de los subsistemas que componen un servicio educativo a distancia, la calidad de la oferta de formación y el impacto deseado en sus receptores (Gergich, Imperatore y Pajoni, 2002).

Tanto el cuerpo docente como los materiales didácticos aparecen como variables dependientes en función de la capacidad que tenga la institución para poder generar equipos de profesionales capacitados y desarrollar una propuesta pedagógica adaptada al medio. La bibliografía, en cambio, está sujeta a una serie de parámetros que son independientes de las organizaciones educativas propulsoras de los sistemas de EAD y que están relacionados con la disponibilidad y accesibilidad a la misma en las condiciones en que hoy lo exige el entorno sobre el que se estructuran estas propuestas, es decir, en un ambiente digital. Claramente, ésta es la tendencia, aunque no siempre es posible cumplir con ella. Los problemas principales derivan de no contar siempre con toda la bibliografía en el soporte y formato deseado y, de obtenerla, todavía resta superar los escollos legales derivados de los derechos de propiedad intelectual.

A priori, son las bibliotecas las encargadas de resolver estos problemas a fin de proveer a los alumnos los 
servicios bibliográficos necesarios para garantizar su formación (Pasch, 2000).

Las bibliotecas y centros de documentación han ejercido siempre un papel central en el desarrollo del aprendizaje en cualquiera de sus niveles y formatos. Dentro del entorno universitario cumplen además un rol fundamental para el ejercicio de la docencia y la investigación. Es importante señalar que en los sistemas educativos no presenciales los servicios de información adquieren una posición central, ya que pasan a ser una puerta de acceso a través de la cual se integran productos y servicios destinados a satisfacer las demandas puntuales de sus alumnos (Pérez, 2000). En los entornos virtuales, en donde hoy conviven la EAD y las bibliotecas, se hace necesaria una relación muy estrecha y un tipo de funcionamiento complementario. En este sentido, desde la bibliotecología y las bibliotecas se han ido diseñando y desarrollando distintos servicios y productos de información que hoy resuelven esta variante y trabajan únicamente en la virtualidad, atendiendo las necesidades y características del usuario on-line (Tramullas, 2004).

Pero la biblioteca en la virtualidad no puede reducirse a un Catálogo Colectivo (Opac) como parte de los servicios de un sistema de $\mathrm{EAD}$, sino que debe integrar un conjunto de herramientas que amplían sus capacidades de cara a satisfacer a un segmento de usuarios con un perfil bien diferenciado, constituyendo lo que algunos investigadores han denominado "bibliotecas híbridas" (Stuart Garcez y Varvakis Rados, 2002). Servicios de novedades de consulta en línea o de referencia, buscadores, secciones de sitios relacionados (creando enlaces entre instituciones calificadas, conocido como Webring), bases de datos de recursos Web, listas de interés, pasarelas temáticas (subject gateway), constituyen solamente algunos ejemplos de las potencialidades del entorno web (Rodríguez Yunta, 2004).

De todos los segmentos educativos, el universitario es el que más ha desarrollado la formación no presencial. En Argentina, esta modalidad ha sido siempre liderada por la universidad pública, comenzando sus primeras experiencias a mediados de la década del ochenta. A pesar de este temprano comienzo, recién a finales de los noventa se sanciona la primer normativa oficial para la instrumentación y gestión de sistemas a distancia en la universidad (Argentina. Ministerio de Educación, 1998). Es importante resaltar en esta resolución ministerial el hecho de que aparezca como uno de los requisitos para el desarrollo de la EAD la existencia de servicios bibliotecarios especializados.

A partir de 1998 la Universidad Nacional de Quilmes* desarrolla el primer campus virtual en la Argentina

${ }^{*}$ http://www.cvq.edu.ar y el primero en su tipo en Latinoamérica, el cual responde a las necesidades de formación continua de diversos sectores sociales diversificados y diseminados geográficamente (Herzog, 2000). Desde entonces, este tipo de oferta educativa no ha cesado de crecer en el país, instalándose como una opción cada vez más atractiva para el inicio o continuación de los estudios universitarios, tanto en el nivel de grado como en el posgrado.

Este desarrollo de la EAD en las universidades argentinas ha estado limitado en muchos casos por la capacidad técnica e infraestructura tecnológica con que se cuenta. En la actualidad no existen estudios que reflejen un diagnóstico integral de esta situación, en cuanto a los niveles de avance de esta modalidad, oferta académica y grado de desarrollo de las tecnologías aplicadas (Knez y Santos, 2002).

Esta investigación se orienta al análisis de uno de los elementos centrales de la EAD señalados: los servicios bibliotecarios en línea. Se indaga sobre el estado e integración de los servicios de información de las bibliotecas en la educación no presencial ofrecida por las universidades públicas argentinas** en entornos virtuales. En este sentido, se pretende establecer un panorama general del nivel de desarrollo y presencia que tiene los servicios bibliotecarios en los proyectos de EAD en curso.

\section{MATERIAL Y MÉTODO}

Esta investigación se basa en un estudio exploratoriodescriptivo de los portales web de las $42^{3}$ universidades públicas argentinas en lo referido a los servicios de EAD que brinda cada una de ellas y los servicios bibliotecarios en línea que se prestan. Los datos fueron recogidos en el periodo mayo-agosto de 2004.

El objetivo del estudio es la observación de los enlaces (links) existentes entre las páginas web dedicadas a la EAD y los OPACs o servicios de información que poseen las universidades y viceversa. Se trata de detectar desde los portales de EAD la presencia o uso de estos servicios bibliográficos y, desde los portales de las respectivas bibliotecas, enlaces o vínculos para los alumnos que acceden desde estos servicios educativos.

\footnotetext{
${ }^{* *}$ No se incluye en este estudio al portal Educar (www.educ.ar) ya que no pertenece a una universidad. Este emprendimiento depende del Ministerio de Educación de la Nación a partir del cual cualquier institución educativa de nivel público puede ofrecer servicios de educación no presencial.

${ }^{3}$ Se incluyen también en este estudio los institutos universitarios dependientes de algunas esferas del Estado.
} 
De alguna manera, a través de este análisis empírico, se pretende demostrar qué rol juegan los servicios bibliotecarios en el nuevo escenario de la EAD a través de la red y la presencia que tienen desde el punto de vista de su importancia en la formación universitaria.

Los datos recogidos son:

- Enlaces detectados desde el/los portal/es de EAD hacia:

- $\quad$ El OPAC (On-line Public Acces Catalog) de la universidad-facultad-departamento

- $\quad$ El OPAC propio

- Un OPAC externo

- Servicios de información externos (bases de datos, revistas electrónicas, e-books, etc.).

- Enlaces detectados desde el OPAC de la universidad hacia:

- El/los portal/es de la EAD.

- Servicios especiales para alumnos de distancia.

A través de los enlaces informativos de cada uno de los servicios de EAD relevados se trata de establecer respecto de la bibliografía:

- El soporte en que se ofrece: papel, digital o ambos.

- La tipología documental que se utiliza: libros, revistas, materiales especiales o general (incluye a todos).

- El nivel de acceso: full o referencial.

También se analizan indicadores generales como:

- Total de la oferta educativa a distancia en las universidades públicas discriminada por:

- Carreras y cursos de grado.

- Carreras y cursos de posgrado.

- Áreas de conocimiento que cubren las ofertas a distancia.

\section{RESULTADOS Y DISCUSIÓN}

La exposición de los resultados se divide en tres segmentos. En el primero se refleja el panorama en cuanto a las características generales de la EAD en las universidades. En el segundo, se muestra la existencia de los servicios bibliotecarios en línea que poseen las instituciones involucradas. En la tercera parte, se analiza la integración y el uso que los sistemas de EAD relevados hacen de estos servicios de información.

El primer dato que surge de esta investigación permite corroborar el importante desarrollo que tienen en la actualidad los planes de EAD implementados desde el sector universitario público en la Argentina. Del total del universo analizado (42 universidades), se desprende que el 46,5\% (20 instituciones) posee servicios educativos a distancia. En total, se contabilizan 119 propuestas distintas entre cursos y carreras. Se observa además que la oferta académica en esta modalidad abarca los dos segmentos tradicionales en la formación universitaria, el grado y el posgrado, con una amplia diversidad de propuestas orientadas a la formación, actualización y perfeccionamiento (gráfico 1).

Con respecto a las áreas disciplinares que cubre este tipo de oferta, se advierte un abanico muy amplio de posibilidades, encontrándose un sesgo muy marcado hacia la especialización en campos específicos y con perfiles netamente interdisciplinarios. En una clasificación realizada ad hoc se desprende que, del total de ofertas relevadas, el $56,7 \%$ proviene de las ciencias sociales y humanas, mientras que el $43,3 \%$ pertenece a las ciencias duras y aplicadas. Mencionaremos como dato anexo que existen tres carreras de grado en el campo de la bibliotecología y documentación.

Una vez delimitada la cantidad y el tipo de oferta de la EAD, el análisis se orienta a establecer el nivel alcanzado en la aplicación de tecnología en el entorno digital, esto es, el grado de virtualidad conseguido en la prestación de los servicios educativos. Para ello se designan tres categorías que guían la observación y permiten clasificar la tipología de propuestas encontradas en la red. Así, la categoría "virtual" agrupa aquellas ofertas que integran todos sus servicios y productos en un ambiente digital accesible en línea, prescindiendo totalmente de la presencialidad o el contacto personal físico. La categoría "semi-virtual" representa aquellos sistemas que prestan gran parte de sus servicios y productos en un entorno digital (módulos didácticos, tutorías, inscripciones) pero que mantienen instancias de presencialidad (evaluaciones, encuentros, etc.). Finalmente, la categoría "no-virtual" para aquellas propuestas de EAD que no utilizan tecnologías derivadas del entorno web o lo hacen de manera muy parcial (como el uso del correo electrónico). Los resultados demuestran que el $79 \%$ de la oferta se presenta en entornos puramente virtuales, mientras que el $16 \%$ se 


\section{GRÁFICO 1}

Tipo de oferta en los planes de EAD en las universidades $(\mathrm{N}=119)$

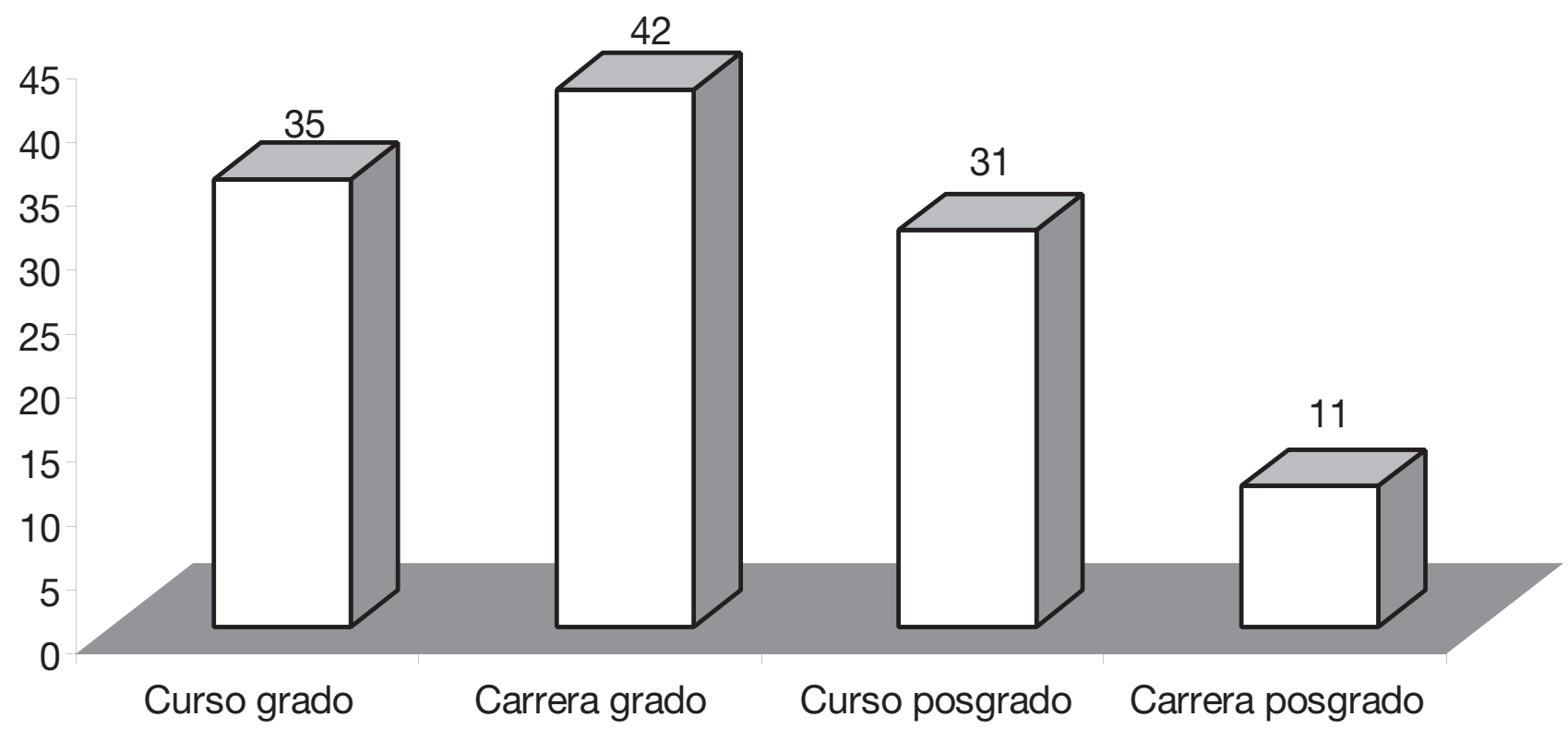

encuentra en un nivel de semi virtualidad. Apenas el $5 \%$ de las propuestas todavía no hacen un uso intensivo de estas tecnologías.

En cuanto a la existencia de los servicios bibliotecarios en línea en las universidades, la observación se orienta a establecer principalmente cuáles de ellas poseen OPACs accesibles desde internet y qué servicios brindan en la consulta a bases de datos externas y material bibliográfico en texto completo. Del total de universidades públicas existentes $(\mathrm{N}=42)$, el 65\% (28 instituciones) posee automatizados sus catálogos y ofrece un servicio de consulta en línea a través de internet de forma centralizada. Entre las universidades que brindan servicios de $\operatorname{EAD}(\mathrm{N}=20)$, el 90\% (18 instituciones) cuenta con OPACs y servicios a otras fuentes de información accesibles a través de la red. Solamente dos instituciones de este último grupo no cuentan con esta posibilidades.

En el tercer nivel de análisis, se intenta establecer la presencia de servicios bibliotecarios integrados a las ofertas de EAD como un elemento destinado a satisfacer y proveer de la bibliografía necesaria para el logro de los objetivos de aprendizaje planteados en el programa. La principal fuente de información para este indicador son los enlaces detectados en los portales de los sistemas a distancia relevados, tal como se expresa en la metodología empleada. El principal inconveniente encontrado es la imposibilidad de ingresar en muchos lugares con acceso restringido, limitado a los alumnos que participan del programa. De todas formas, para complementar la información obtenida, se recurre a los enlaces informativos que generalmente poseen estos sitios, páginas de las programas de estudio en donde se explica el modo en que se obtendrá la bibliografía y, en algunos casos, a las demostraciones disponibles para posibles interesados.

Del total de los programas de EAD $(\mathrm{N}=119)$, solamente el 24,5\% (29) brinda acceso a un OPAC propio, diseñado para satisfacer las demandas de sus usuarios específicos. El resto de los programas $(75,5 \%)$ cumple con los requisitos de acceso a la bibliografía ofreciendo enlaces a módulos de estudio recopilados por los docentes o coordinadores para tal fin, a OPACs existentes en la propia institución o fuera de la misma (OPACs externos), a bases de datos y revistas de acceso gratuito o enlaces a documentos almacenados en la web.

En el análisis de este segundo grupo de programas, se observa que una buena parte de ellos no utiliza los servicios bibliotecarios ya existentes en las unidades académicas de donde dependen, por lo general facultades o departamentos. Así, se detecta que el $32 \%$ de las propuestas a distancia no hace uso (no poseen enlaces o menciones) de los OPACs accesibles desde las páginas alojadas en los sitios de donde dependen ambos servicios (el de distancia y el bibliotecario). En algunos casos (5\%), se llega al extremo de la coexistencia de dos servicios bibliotecarios paralelos, uno ofrecido por el sistema de EAD y otro dependiente de la facultad, sin que entre ambos medie relación alguna.

En cuanto al soporte y tipología del material bibliográfico se detecta que solamente el 15\% del total de programas de EAD relevados utilizan material impreso (papel), 


\section{GRÁFICO 2}

Disponibilidad de la bibliografía utilizada por los programas de EAD $(\mathrm{N}=119)$

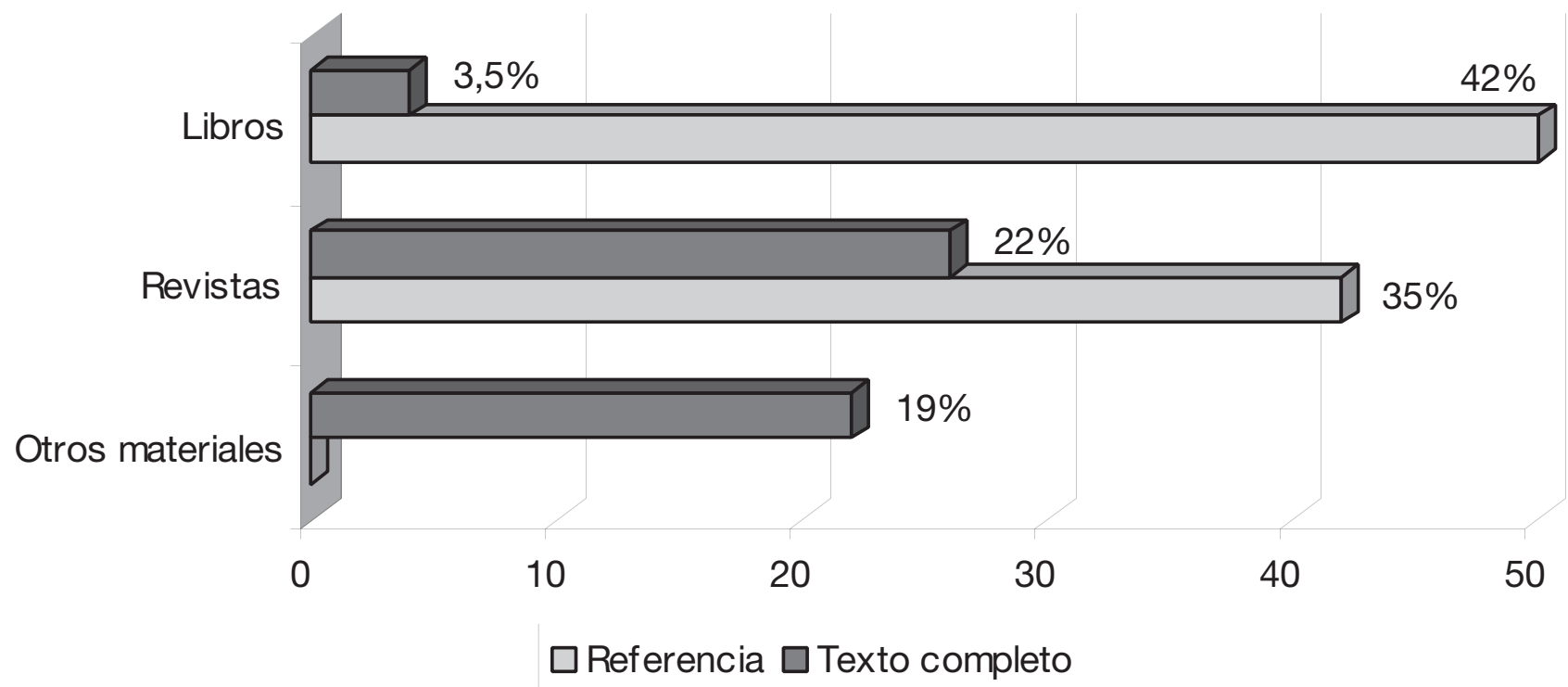

mientras que el resto (85\%) permite un acceso a la referencia o al documento completo en soporte digital de acuerdo con el gráfico 2.

Puede observarse que uno de los recursos más utilizados en texto completo son las revistas, guardando una lógica con la disponibilidad que tiene este tipo de fuentes en formato electrónico en la red, tanto sea a través de suscripciones como en forma gratuita. En este segmento cobran mucha importancia las publicaciones académicas que aplican la filosofía Open Acces. En segundo lugar aparece la tipología "otros materiales" totalmente accesible en soporte digital, dado que se trata en su mayoría de literatura producida por los propios docentes bajo la forma de apuntes de cátedra, informes, revisiones bibliográficas, etc. Los libros, como era esperable, muestran un porcentaje muy bajo en cuanto a la posibilidad de consultarlos en línea a texto completo, sobre todo porque son obras muy especializadas y cuyas ediciones datan de muchos años.

Finalmente fue posible comprobar que las carreras y cursos provenientes de las ciencias duras y aplicadas tienen menos obstáculos para el acceso a la bibliografía en soporte digital, ya que utilizan mucho las publicaciones periódicas e informes técnicos. Por el contrario, en las sociales y humanas existe un fuerte predominio del libro y, por consiguiente, mayores restricciones para su utilización en sistemas a distancia, recurriendo a la opción del diseño y desarrollo de manuales de cátedra por los propios docentes o académicos contratados para tal efecto.

Esta última alternativa se ha transformado en al actualidad en una fuerte tendencia no solamente por sus costos, sino además porque garantiza su libre acceso. En la actualidad el Massachusetts Institute of Technology (MIT) desarrolla el proyecto OpenCourseWare (MIT OCW)* orientado a promover una estandarización en la producción de este tipo de materiales electrónicos a la vez de convertirse en una iniciativa para garantizar el acceso gratuito a los mismos.

\section{CONCLUSIONES}

A través del estudio realizado se ha podido mostrar que la EAD en las universidades públicas argentinas tiene un importante desarrollo y que además buena parte de los proyectos en curso utilizan plenamente a internet como espacio de interacción para la oferta de sus servicios educativos. Esta realidad se encuentra a tono con las tendencias internacionales, sobre todo en los aspectos relativos a la aplicación de las últimas tecnologías aparecidas para la gestión de estos sistemas.

La contracara mostrada en esta investigación es la notable ausencia de los servicios bibliotecarios como actores necesarios e indispensables en la planificación de una oferta educativa a distancia. Como se ha demostrado, es muy bajo el porcentaje de propuestas que cuentan entre sus prestaciones con un servicio de información ajustado a las pautas que rigen esta modalidad de formación.

Se puede observar que en el universo estudiado tanto los servicios bibliotecarios como los de la EAD en entornos virtuales funcionan, en la mayoría de los casos, como mundos paralelos, sin conexión.

\footnotetext{
" http://www.mit.edu/opencouseware/
} 


\section{Gustavo Liberatore / Andrés Vuotto}

Una primer conclusión derivada de esta última característica es que los sistemas de EAD de la universidades públicas argentinas se encuentran, en términos generales, en una etapa adolescente en el sentido de no contar con todos los elementos necesarios para alcanzar los niveles de calidad que permitan consolidar una oferta educativa integral.

Desde un enfoque profesional bibliotecario, es evidente que la biblioteca, a pesar de encontrarse en pleno proceso de virtualización en el ciberespacio argentino, no ha podido todavía posicionarse como un producto necesario en los procesos de enseñanza-aprendizaje en los planes de la EAD universitaria. En apariencia, la biblioteca y el profesional de la información se encuentran ausentes en la formulación y concepción de los proyectos pedagógicos para entornos virtuales. De alguna manera, la biblioteca no ha podido trasladar todavía al ambiente digital la imagen y prestigio que goza en el mundo físico.

Esto constituye un desafío para el mundo profesional bibliotecario desde el punto de vista de la integración a estas nuevas modalidades educativas a partir del diseño de productos y servicios ajustados a las necesidades de este entorno. El alumno de un sistema a distancia, el usuario potencial, posee rasgos particulares que la biblioteca deberá atender: soledad en su situación de aprendizaje, incertidumbre ante lo nuevo, necesidad de una reafirmación constante en la confianza en el sistema, auxilio en momentos críticos e información confiable y a tiempo para la toma de decisiones en los procesos de aprendizaje.

Para transformar este escenario se hace necesario generar iniciativas a través de esfuerzos conjuntos y una toma de conciencia tanto desde los sectores bibliotecarios de las instituciones como de las coordinaciones académicas y organismos de gobierno de las universidades que tienen el poder y el compromiso de crear los espacios de trabajo (tanto políticos cómo físicos) adecuados a estas realidades.

Artigo recebido em 26-10-2004 e aceito para publicação em 29-03-2005.

\section{REFERENCIAS}

ARGENTINA. MINISTERIO DE EDUCACIÓN, CIENCIA Y TECNOLOGÍA. DIR. NAC. DE GESTIÓN UNIVERSITARIA. Orientación instrumental para la presentación y seguimiento de acciones del nivel universitario con gestión a distancia: resolución 1716/98. Disponível em: <http://www.cin.edu.ar/>. Acesso em: abr. 2004.

BECERRA, MARTÍN A. Apuntes sobre educación superior y virtualidad en la Argentina. In: FLORES, Jorge; BECERRA, Martín (Comp.). La educación superior en entornos virtuales. Bernal : Univ. Nac. de Quilmes, 2002. p. 27-50.
COOKSON, PETER S. Access and euity in distance education: research and development, and quality concerns. Revista Electrónica de Investigación Educativa, v. 4, n. 2, 2002. Disponível em: < http://redie.ens.uabc.mx/ vol4no2/contenido-cookson.html>. Acesso em: abr. 2004.

GERGICH, M,; IMPERATORE, A.; PAJONI, H. Entre la letra y la pantalla: la trama y el lugar de los materiales didácticos en el programa UVQ. In: FLORES, Jorge; BECERRA, Martín (Comp.). La educación superior en entornos virtuales. Bernal : Univ. Nac. de Quilmes, 2002. p. 295-307.

HERZOG, ROMAN. Las nuevas tecnologías de información y comunicación en la enseñanza y la ciencia: experiencias de Argentina. Hamburgo : Instituto de Estudios Americanos, 2000.

KNEZ, LILIAN ANA; SANTOS SOUZA, ALEJANDRA. Una mirada a la normativa de la educación no presencial en la Argentina: el caso de la educación en entornos virtuales de aprendizaje. In: FLORES, Jorge; BECERRA, Martín (Comp.). La educación superior en entornos virtuales. Bernal : Univ. Nac. de Quilmes, 2002. p. 151-165.

LEVIS, DIEGO; GUTIÉRREZ FERRER, MARÍA LUISA. ¿Hacia la herramienta educativa universal? Educar y aprender en tiempos de Internet. Buenos Aires : CICCUS/La Crujía, 2000. 171 p.

MASSACHUSETTS INSTITUTE OF TECHNOLOGY. Open course ware. Disponível em: <http://www.mit.edu/opencouseware/>. Acesso em: abr. 2004.

PAGÉS SANTACANA, ANNA. Universidades virtuales: el caso de la Universidad Oberta de Catalunya. Edutec, Revista Electrónica de Tecnología Educativa, n. 14, 2001. Disponível em: < http://www.uib.es/depart/gte/ edutec-e/revelec15/albert_sangra.htm>. Acesso em: abr. 2004.

PASCH, Grete. La biblioteca digital soporte de la educación a distancia. Disponível em: <www.gslis.utexas.edu/ lis $312 \mathrm{~g}>$. Acesso em: maio 2004.

PÉREZ, ADORACIÓ. Bibliotecas y centros de documentación en un entorno virtual. In: DUART, Joseph; SANGRÁ, Albert (Comp.). Aprender en la virtualidad. Barcelona : Gedisa, 2000. p. 223-240.

RED UNIVERSITARIA DE EDUCACIÓN A DISTANCIA. Programas y proyectos de educación superior. [S. L.), 2002. Disponível em: <http:// www.virtual.unlar.edu.ar/RUEDA/>. Acesso em: abr. 2004.

RODRÍGUEZ YUNTA, LUIS. Pasarelas temáticas en Internet: un modelo de directorio basado en la aplicación de técnicas documentales. Hipertext.net, n. 2, 2004. Disponível em: <http://www.hipertext.net>. Acesso em: set. 2004.

SANGRÁ MORER, Albert. Educación a distancia, educación presencial y usos de la tecnología: una tríada para el progreso educativo. Edutec, Revista Electrónica de Tecnología Educativa, n. 15, 2002. Disponível em: $<$ http://www.uib.es/depart/gte/edutec-e/revelec15/albert_sangra.htm>. Acesso em: abr. 2004.

SILVIO, JOSÉ. La virtualización de la educación superior: alcances, posibilidades y limitaciones. Revista Educación Superior y Sociedad, v. 2, n. 1, p. 34- 45, 1998.

STUART GARCEZ, ELIANE; VARVAKIS RADOS, GREGÓRIO J. Biblioteca híbrida: um novo enfoque no suporte à educação a distância. Ciencia da Informnação, v. 31, n. 2, p. 44-51, 2002.

TRAMULLAS SAZ, JESÚS. El diseño centrado en el usuario para la creación de productos y servicios de información digital. Forinfo: Revista Iberoamericana de Usuarios de Información, n. 22/23, p. 12, 2004.

ZAPATA, MIGUEL. Sistemas de educación a distancia a través de redes: rasgos para la propuesta de evaluación de la calidad. Revista de Educación a Distancia, n. 9, 2003. Disponível em: <http://www.um.es/ead/red/>. Acesso em: mar. 2004. 